\title{
ARTÍCULOS
}

\section{Crecimiento de una plantación joven en fajas con especies nativas en la Cordillera de Los Andes de la provincia de Valdivia}

\author{
Growth of a young native species strip plantation in the Valdivian Andes, Chile.
}

\author{
Claudio Alvarez Pa*, Antonio Lara ${ }^{b}$ \\ *Autor de correspondencia: aUniversidad Austral de Chile, Instituto de Silvicultura, Laboratorio de Dendrocronología, Valdivia, \\ Chile, claudioalvarez7@yahoo.es \\ bUniversidad Austral de Chile, Valdivia, Chile.
}

\begin{abstract}
SUMMARY
The goal of this study was to assess the growth of a four year-old Nothofagus dombeyi, N. alpina and Gevuina avellana plantation in San Pablo de Tregua, in the Valdivian Andes at c. $650 \mathrm{~m}$ of elevation. The plantation was established in eight strips 8-11 meter wide opened on a Chusquea culeou thicket, a native bamboo species that has undergone massive die back after a mast flowering event in 2001. Strips 1 through 6 were 37-74 m long, while strips 7 and 8 were 174 and 196 m long, respectively. Old-growth forests formed the upper edge of the strips, while the lower edge was open grassland. Strips were separated by 7-10 meters of untreated thicket, dominated by a dense mass of dead standing Chusquea culms with a height of 8-11 m. Mean annual increment (MAI) in height and diameter at ground height were analyzed as a function of cover and distance to the untreated thicket between strips and to the forest edge. $N$. dombeyi survival and MAI in height and diameter were higher at lower cover of the thicket between strips. $N$. alpina showed higher diameter MAI when the cover of the thicket was $<25 \%$. This study concluded that plantations in strips for $N$. dombeyi that were tested show adequate survival and initial growth for ecologic restoration purposes in forests that have been logged and replaced by Chusquea thickets.
\end{abstract}

Key words: strip plantations, Nothofagus dombeyi, N. alpina, Gevuina avellana

\section{RESUMEN}

El objetivo de esta investigación fue evaluar el crecimiento de una plantación en fajas de cuatro años de edad con las especies Nothofagus dombeyi (coigüe), N. alpina (raulí) y Gevuina avellana (avellano). El estudio se realizó en el fundo San Pablo de Tregua, ubicado en la Cordillera de Los Andes de la provincia de Valdivia, en ocho fajas de plantación confeccionadas después de una floración y muerte masiva ocurrida en un matorral de Chusquea culeou el año 2001. Las fajas 1 a 6 presentaron longitudes de 37 a 74 m; y las fajas 7 y 8, 174 a 196 m, respectivamente. Los anchos de faja comprendieron 8 a 11 m. Mediante análisis de covarianza, de varianza y prueba t de Student se analizó el incremento medio anual (IMA) en altura y diámetro de cuello respecto de los factores: grado de cobertura de la interfaja, distancia a la misma y distancia al borde del bosque. Coigüe registró una sobrevivencia mayor al $90 \%$ en todas las fajas y presentó un mayor IMA en altura y diámetro de cuello en condiciones de mayor exposición al sol $(P<0,05)$. Raulí presentó diferencias significativas sólo en el IMA en diámetro de cuello creciendo más las plantas con una cobertura de interfaja menor a 25\% $(P<0,05)$. Se concluyó que las plantaciones de coigüe en fajas resultaron adecuadas para su desarrollo inicial con objetivos de restauración ecológica, en bosques del tipo Coigüe-Raulí-Tepa degradados por tala con fines madereros.

Palabras clave: plantación en fajas, Nothofagus dombeyi, N. alpina, Gevuina avellana.

\section{INTRODUCCIÓN}

El aprovechamiento histórico del bosque nativo sin un adecuado manejo ha provocado el establecimiento de especies arbustivas pioneras como Chusquea culeou Desv. (colihue), las cuales forman densas poblaciones que retardan e inhiben la regeneración de plantas arbóreas nativas. Es por esto que la corta en fajas de tales formaciones y la plantación con especies nativas es una buena alternativa para la restauración ecológica y productiva de los bosques nativos. La restauración y enriquecimiento de estos ecosistemas forestales alterados, a una escala regional, tendrían impacto social, económico y ambiental muy positivo, ya que incorporaría extensas superficies alteradas y de baja productividad al manejo forestal nacional (González et al. 1997). Para lograr una recuperación productiva se necesita 
evaluar distintas técnicas de recuperación, así como también conocer las potencialidades de crecimiento de diferentes especies arbóreas que sean una alternativa en la repoblación de áreas con bosques degradados.

Entre las técnicas de recuperación se encuentra el enriquecimiento de bosques alterados a través de plantaciones a campo abierto, en claros o bien en fajas. La plantación en fajas consta de un diseño longitudinal, de ancho variable, paralelas entre sí, previamente despejadas de vegetación, abiertas al interior del bosque y donde los ejemplares se plantan en una o más hileras (Hernández y Vita 2004). Esta técnica de recuperación presenta ventajas con respecto a la de campo abierto: por ejemplo, reduce los costos iniciales debido a una silvicultura menos intensiva que involucra actividades culturales sólo en una porción de la superficie total intervenida. Además, este tipo de diseño tiene un efecto en el desarrollo de las plantas, producto de factores ecológicos que se relacionan con el tipo y grado de protección lateral que ofrece la vegetación remanente entre las fajas (interfajas). Esta protección permite mantener en cierta medida las condiciones ambientales del bosque original, las que varían de acuerdo al ancho de las fajas (Otero et al. 1994). Asimismo, las temperaturas máximas del aire pueden ser superiores en condiciones de campo abierto comparadas con un bosque bajo cortas de protección, durante las estaciones de primavera y verano (Barg y Edmonds 1999) lo que es importante para la absorción de agua y nutrientes y la respiración de las raíces (Weinberger y Ramírez 2001).

Por otra parte, es importante considerar la heterogeneidad en la disposición y tipo de vegetación ubicada entre las fajas, ya que genera una gran diversidad de condiciones locales de cobertura y estratificación vertical, lo que se traduce en múltiples tipos de microhábitat. Éstos se ven influenciados por cambios a nivel de terreno como variaciones en la exposición, pendiente, microrrelieve y ubicación fisiográfica, y en el tipo de cobertura (árbol, arbusto), características de la copa (densidad, persistencia), altura de inicio de la copa desde el suelo y ubicación de la planta en relación al ejemplar que le proporciona protección o competencia (Hernández y Vita 2004).

En el presente trabajo se evalúan dos especies del género Nothofagus: N. dombeyi (Mirb.) Oerst. (coigüe) y N. alpina (P. et E.) Oerst. (raulí), las que concitan el mayor interés desde el punto de vista silvicultural (Donoso et al. 1999) y son consideradas las de mayor potencial productivo en Chile. Además, se evalúa el crecimiento de Gevuina avellana Mol. (avellano) que no es una especie de rápido crecimiento como las anteriores, sin embargo, representa una alternativa para el repoblamiento de bosques alterados debido a su destacado valor como especie ornamental y productora de frutos (Tacón y Palma 2006).

Este trabajo tiene como objetivo general evaluar el desarrollo de una plantación en fajas de cuatro años de edad con las especies coigüe, raulí y avellano, ubicada en la Cordillera de Los Andes y establecida luego de la corta de una densa población de colihue seco que dominaba el área. Los objetivos específicos son evaluar: a) la sobrevivencia de las plantas de las tres especies; b) la calidad de las plantas en relación a los factores grado de cobertura vegetal de interfaja, distancia a la interfaja y distancia al borde del bosque, y c) los crecimientos en altura y diámetro de cuello de las plantas derivados de la posición espacial con respecto al grado de cobertura vegetal, distancia a la interfaja y distancia al borde del bosque.

\section{MÉTODOS}

Caracterización del área de estudio. El estudio se realizó en el predio San Pablo de Tregua de propiedad de la Universidad Austral de Chile, ubicado en la comuna de Panguipulli, provincia de Valdivia, de coordenadas $39^{\circ} 30^{\prime} \mathrm{S}, 72^{\circ} 02^{\prime} \mathrm{O}$, con una altitud aproximada de 650 m s.n.m. Los bosques naturales pertenecen al tipo forestal Coigüe-Raulí-Tepa (Donoso 1981a). La plantación se ubica en una ladera de exposición $210^{\circ} \mathrm{SO}$ con pendientes que fluctúan entre $3^{\circ}$ y $14^{\circ}$. El clima del lugar se caracteriza por una alta precipitación anual, veranos muy cortos y secos, e inviernos húmedos. Se clasifica en el sistema Köppen como un clima oceánico templado, con una suave influencia mediterránea. La temperatura anual promedio es de $11^{\circ} \mathrm{C}$, la temperatura mínima promedio del mes más frío (agosto) es $5^{\circ} \mathrm{C}$, la máxima promedio del mes más cálido (febrero) es $20^{\circ} \mathrm{C}$ y anualmente precipitan 4.000 a $5.000 \mathrm{~mm}$. El número de heladas al año varía entre 30 y 50, y en invierno se producen comúnmente precipitaciones sólidas (nieve) (Lara et al. 2002). Los suelos son derivados de cenizas volcánicas modernas, correspondientes a la serie Liquiñe (CIREN 2001), los que son moderadamente profundos a profundos y presentan textura superficial franco arenosa fina y color pardo oscuro en el matiz 7.5 YR en profundidad. El sustrato está constituido por pómez fuertemente meteorizada. Posee alto contenido de gravas pumicitas y presenta drenaje bueno a excesivo (Mella y Kühne 1985). Además, presenta densidad aparente baja $\left(<0,6 \mathrm{~g} \mathrm{~cm}^{-3}\right)$ y muy alta capacidad de agua aprovechable $(>250 \mathrm{~mm}$ en $1 \mathrm{~m}$ de profundidad) (Donoso et al. 2007).

Condición inicial de la plantación. La plantación fue realizada el año 2001 a principios del mes de agosto y para su establecimiento se construyeron fajas con anchos de 8 a $11 \mathrm{~m}$, en el sentido de la pendiente y en forma paralela entre sí. Las ocho fajas de plantación estudiadas poseen una superficie total aproximada de 1,3 hectáreas, de las cuales 0,6 corresponden a la superficie efectivamente plantada con especies nativas y el resto corresponde a la zona ubicada entre fajas (interfajas). Las interfajas comprendieron anchos de 7 a $10 \mathrm{~m}$, dominadas por colihue seco y rebrotes de la misma especie que colonizaron estas zonas después de una floración y muerte masiva de colihues ocurrida entre diciembre del año 2000 y mayo de 2001 (figura 1). Al 
establecer la plantación se llevaron a cabo labores de corta de colihue seco, ordenando los desechos en pilas a los lados de cada interfaja. Todas las fajas presentaron cuatro hileras de plantación, con un esquema de $2 \times 2 \mathrm{~m}$ a $3 \times 3 \mathrm{~m}$. Las hileras laterales fueron ubicadas a un metro de la interfaja de colihue (figura 2). En la faja 1 se establecieron cuatro hileras de plantación con la especie coigüe. En la faja 2 la hilera 1 (al oriente) se estableció de manera mixta con coigüe y avellano, las hileras centrales (hileras 2 y 3 ) con coigüe, y la hilera 4 (al poniente) con avellano. Las fajas 3 a 6 presentaron hileras laterales de avellano y centrales de coigüe. En la faja 7 se estableció de manera mixta en las hileras laterales avellano y raulí, y en las centrales coigüe. En la faja 8 las hileras laterales presentaban raulí y las centrales coigüe. En todas las fajas se encontraron árboles remanentes de las especies Laureliopsis philippiana (Looser) Schodde (tepa) de 5 a $10 \mathrm{~m}$ de altura, y en menor cantidad Saxegothaea conspicua Lindl. (mañío de hojas cortas) con un rango de altura entre 15 y $26 \mathrm{~m}$.

Las fajas 1 a 6 presentaron una protección lateral otorgada por la cobertura de interfajas, principalmente de colihue (seco y verde). Tenían una longitud de 37 a $74 \mathrm{~m}$ y una pendiente de $12^{\circ}$ a $14^{\circ}$. Las fajas 7 y 8 , además de

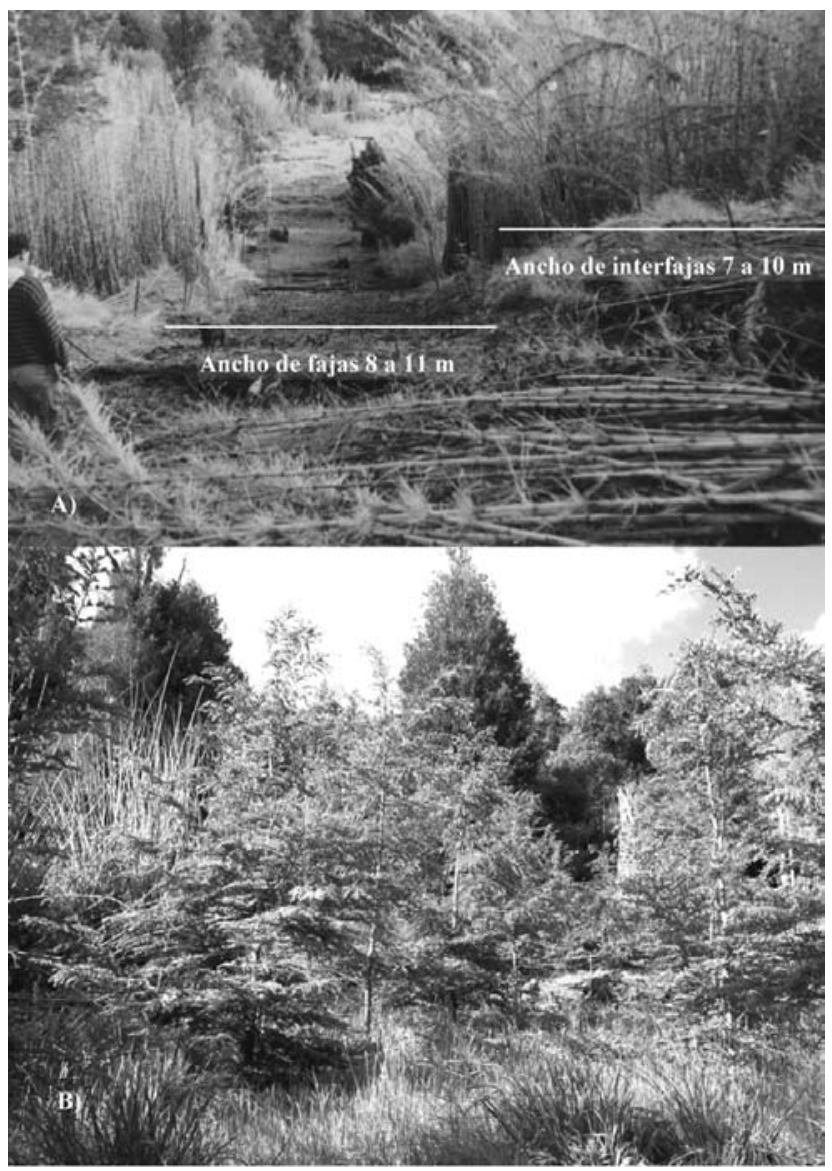

Figura 1. Vista panorámica de fajas: A) año 2001, B) año 2005.

Panoramic view of the strips a) 2001, b) 2005 .

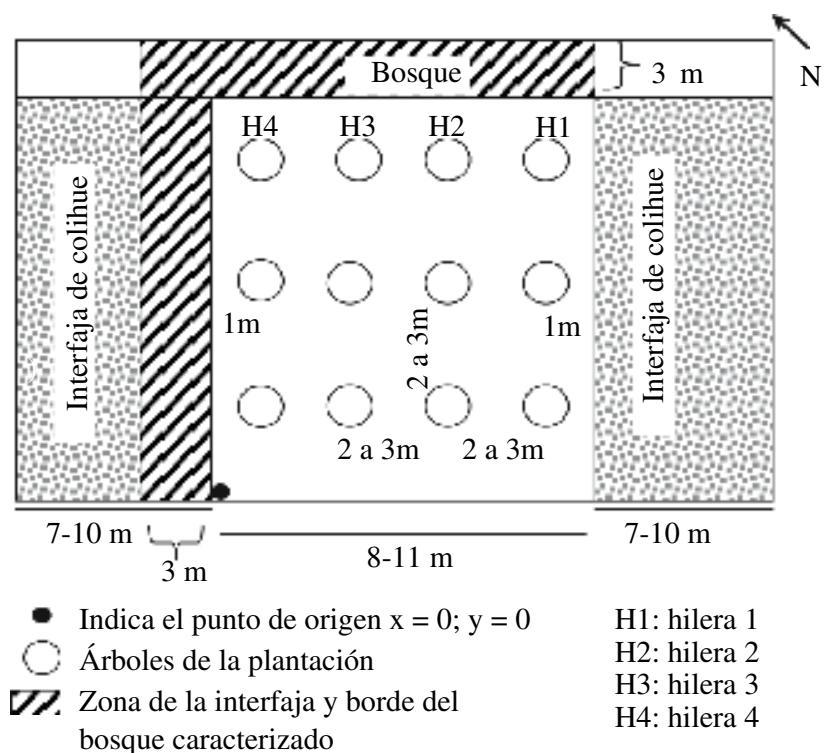

Figura 2. Esquema de la plantación, zona de interfaja y borde del bosque caracterizado.

Schematic representation of the plantation and the area of the Chusquea thicket between strips and to the forest edge that were analyzed.

la protección lateral de colihue, presentaron una protección vertical dada por las copas de árboles remanentes tanto en las fajas de plantación como en las interfajas; tenían una longitud de 174 y $196 \mathrm{~m}$, respectivamente, y un rango de pendientes de $3^{\circ}$ a $14^{\circ}$ (cuadro 1 ).

Antecedentes de la plantación. Las plantas de coigüe fueron cultivadas en vivero a raíz desnuda y la procedencia de las semillas fue la Cordillera de Los Andes de la provincia de Valdivia. Las plantas de raulí fueron cultivadas en contenedores y las de avellano a raíz desnuda. Antecedentes sobre la procedencia de las semillas de raulí y avellano no se registraron en la época de establecimiento de la plantación. Las plantas de las tres especies fueron del tipo 2/0. Los tratamientos para evitar la competencia de especies arbustivas y herbáceas en la plantación fueron el desbroce manual, realizado en el verano del año 2003, y la aplicación del herbicida Galant plus en una dosis de $3 \mathrm{~L} \mathrm{ha}^{-1}$, en el verano del año 2005.

Recolección de datos. Se realizó un censo a las ocho fajas de plantación en los meses de agosto y octubre de 2005, considerando las variables: especie, diámetro a la altura del cuello (DAC), altura total, sanidad y forma; también se determinó la sobrevivencia como la relación porcentual del número de plantas vivas por especie al inicio (año 2001) y final (año 2005). Para determinar la posición espacial de las plantas y de los árboles remanentes presentes en cada una de las fajas, se extendió una huincha de distancia en el sentido longitudinal entre las hileras 1 y 2 (posteriormente 3 y 4 ) y otra en sentido transversal 
Cuadro 1. Características de las fajas evaluadas y número de árboles por especie en cada faja.

Main features of the strips and number of trees per species in each strip.

\begin{tabular}{|c|c|c|c|c|c|c|c|c|c|}
\hline \multirow{2}{*}{$\begin{array}{l}\text { Faja } \\
\mathrm{N}^{\circ}\end{array}$} & \multirow{2}{*}{$\begin{array}{l}\text { Longitud } \\
\text { (m) }\end{array}$} & \multirow{2}{*}{$\begin{array}{l}\text { Ancho } \\
\text { (m) }\end{array}$} & \multirow{2}{*}{ Pendiente $\left({ }^{\circ}\right)$} & \multirow{2}{*}{$\begin{array}{l}\text { Área } \\
\text { (ha) }\end{array}$} & \multicolumn{2}{|c|}{$\mathrm{AB}\left(\mathrm{m}^{2} \mathrm{ha}^{-1}\right)$} & \multicolumn{3}{|c|}{$\mathrm{N}^{\circ}$ de plantas por faja } \\
\hline & & & & & Faja $^{1}$ & Interfaja $^{2}$ & Coigüe & Avellano & Raulí \\
\hline 1 & 50 & 8 & 13 & 0,04 & 0,20 & 0,09 & 74 & - & - \\
\hline 2 & 74 & 11 & 14 & 0,08 & 0,86 & 0,63 & 82 & 10 & - \\
\hline 3 & 65 & 9 & 12 & 0,05 & 0,05 & 3,62 & 47 & 8 & - \\
\hline 4 & 50 & 9 & 12 & 0,04 & 0,01 & 0,27 & 37 & 14 & - \\
\hline 5 & 52 & 9 & 13 & 0,04 & 2,63 & 0,25 & 30 & 17 & - \\
\hline 6 & 37 & 8 & 14 & 0,02 & 0,01 & 1,81 & 23 & 20 & - \\
\hline 7 & 174 & 9 & $14,8,3^{3}$ & 0,15 & 0,28 & 5,33 & 125 & 26 & 42 \\
\hline 8 & 196 & 9 & $13,8,3^{3}$ & 0,17 & 5,73 & 5,67 & 89 & - & 57 \\
\hline
\end{tabular}

${ }^{1} \mathrm{AB}$ : área basal de árboles remanentes en las fajas. ${ }^{2}$ Área basal de árboles remanentes en las interfajas. ${ }^{3}$ Zonas alta, media y baja, respectivamente. - Indica que no se encontraban plantas de la especie.

tomando como origen (coordenadas $\mathrm{x}=0 \mathrm{e} \mathrm{y}=0$ ) el borde inferior noroeste de la faja de plantación. Esto permitió generar un plano identificando la especie, la distancia a la interfaja y la distancia al borde del bosque (figura 2). La posición espacial de cada una de las plantas respecto al punto de origen fue importante para determinar el efecto en la calidad y crecimiento de las plantas, de acuerdo a la incidencia del sol que éstas presentaban en relación al grado de cobertura de vegetación de interfaja, distancia a la interfaja y distancia al borde del bosque.

Caracterización de interfajas y borde del bosque. Se caracterizó la interfaja a través de un transecto longitudinal, considerando tres metros desde el borde noroeste de la faja hacia el interior de la interfaja. En tanto, el borde del bosque se caracterizó a través de un transecto transversal de tres metros de ancho, desde el borde superior de la faja hacia el interior del bosque (figura 2). En ambos casos se midieron las variables: especie, diámetro a la altura del pecho (DAP) y altura total de los árboles remanentes.

Determinación del grado de cobertura. En la zona de interfaja y borde de bosque se estimó la cobertura del estrato arbustivo y arbóreo, a través de cuatro categorías $(1=0$ a $25 \% ; 2=25$ a $50 \% ; 3=50$ a $75 \%$ y $4=75$ a $100 \%)$. Además, se registró la altura promedio de los colihues verdes y secos. El registro del grado de cobertura de la vegetación de interfaja se determinó tomando el mismo punto de origen empleado para la generación del plano con la ubicación espacial de las plantas en las fajas de plantación, entonces el borde de interfaja se localizó a la izquierda de cada una de las fajas evaluadas (figura 2). De este modo, se determinó el grado de cobertura de la vegetación de interfaja y, por lo tanto, la proyección de sombra que incidía en cada una de las plantas evaluadas.

Calidad de las plantas. La calidad de las plantas se obtuvo a través de la combinación de categorías de sanidad y forma. Las categorías de sanidad se definieron en sanidad 1: plantas sin daño aparente; sanidad 2: plantas hasta la mitad de la copa clorótica o dañada por heladas; sanidad 3: plantas con más de la mitad de la copa clorótica o dañada por heladas, y sanidad 4: plantas quebradas por la nieve. Las categorías de forma se definieron en forma 1: fuste recto y sin bifurcaciones, ápice bien diferenciado; forma 2: con ápice seco o sin ápice dominante, doble flecha, y forma 3: bifurcaciones fuertes, más de tres ápices principales. Finalmente las categorías de calidad se definieron en calidad 1: sanidad y forma 1; calidad 2: sanidad o forma 2; calidad 3: sanidad o forma 3, y calidad 4: sanidad o forma 4.

Procesamiento y análisis de los datos. Se utilizaron los datos registrados en noviembre del año 2001 al cabo de tres meses de efectuada la plantación. Estos antecedentes consideraron mediciones de las variables especie, altura y estado sanitario para todas las plantas. Además, se consideró una nueva toma de datos llevada a cabo en los meses de agosto y octubre del año 2005. La variable incremento medio anual (IMA) en altura de las plantas fue definida como la diferencia entre la altura total en el año 2005 y la altura registrada en el año 2001, dividido por los cuatro periodos vegetativos que tenía la plantación. La variable IMA en diámetro de cuello fue definida como el cuociente entre el diámetro de cuello total dividido por los cuatro periodos vegetativos, ya que no se registraron mediciones de esta variable en el año 2001. Mediante análisis de covarianza 
se determinó si existían diferencias en el IMA en altura en relación a los factores considerados para este estudio. Este análisis se realizó por ser el más adecuado, ya que considera ajustar el efecto de la altura inicial (año 2001). En el caso del diámetro de cuello se utilizó prueba $t$ de Student y análisis de varianza (ANDEVA) de una vía para determinar diferencias en el crecimiento, debido a que no se contaba con valores iniciales de la variable diámetro de cuello. Cuando se presentó un ANDEVA significativo, se utilizó en una etapa posterior la prueba de comparaciones múltiples de Tukey para identificar las medias con diferencias significativas.

El factor distancia al borde del bosque se definió como las plantas establecidas a más o a menos de $30 \mathrm{~m}$ del borde del bosque en las fajas 1 a 6 . Para las fajas 7 y 8 la distancia al borde del bosque se definió según zona de establecimiento, nombrándose como zona alta a aquellas plantas establecidas a menos de $60 \mathrm{~m}$ del borde del bosque; zona media a plantas establecidas entre $60 \mathrm{y}$ $120 \mathrm{~m}$ del borde del bosque y zona baja a más de $120 \mathrm{~m}$ del borde del bosque. Ello a fin de determinar si existen diferencias significativas en el crecimiento, asociadas a las características de micrositio que presentan cada una de estas zonas. En el análisis de la calidad de las plantas se utilizó la prueba no paramétrica Kruskal-Wallis. Todas las pruebas se hicieron con un nivel de significancia del $95 \%$.

Con el objetivo de determinar si existía un patrón espacial en los mayores IMA en altura y diámetro de cuello de las plantas, se les representó gráficamente utilizando el programa Surfer para Windows (Golden Software Inc. 1999). A partir de la información del censo realizado en las fajas se confeccionó una gráfica en dos dimensiones y el método Kriging de interpolación para la presentación de los crecimientos. La interpretación de los resultados fue por faja y sólo las especies coigüe y raulí fueron analizadas a través de este procedimiento, ya que avellano presentó un número bajo de individuos vivos, lo que dificulta el análisis e interpretación de la distribución espacial del crecimiento.

\section{RESULTADOS}

Descripción de las interfajas. La composición de la vegetación de interfajas presentó en su gran mayoría plantas de colihue seco y verde, con un rango de altura entre 7 y $10 \mathrm{~m}$; árboles remanentes principalmente de Myrceugenia planipes (Hooker et Arnott) Berg (patagua), Laureliopsis philippiana (tepa) en las interfajas 1 a 6 y ejemplares de esta última especie, junto a mañío de hojas cortas (Saxegothaea conspicua) de grandes dimensiones, en las interfajas 7 y 8. En el estrato arbustivo y herbáceo se encontraron las especies Azara lanceolata Hook. f. (corcolén), Campsidium valdivianum (Phil.) Skottsb. (voqui), Ribes magellanicum Poir. sbsp. magellanicum (zarzaparrilla), Acaena ovalifolia R. et P. (cadillo), Osmorhiza chilensis H. et A. (asta de cabra), Digitalis purpurea L. (cartucho), Megalastrum spectabile (Kaulf.) A. R. Sm. et R. C. Moran var. spectabile (helecho) y Loasa acanthifolia Desr.

Cobertura de interfajas y borde del bosque. Los valores medios de cobertura permitieron identificar las interfajas con cobertura media similar. La interfaja 1 obtuvo una media inferior a $30 \%$, las interfajas 2 a la 7 presentaron valores medios entre 30 y $60 \%$, y la interfaja 8 un valor medio mayor a $60 \%$ (figura 3). El borde del bosque que limita con la parte superior de las fajas presentaba una cobertura de copas mayor a $75 \%$ y alturas entre 20 y $30 \mathrm{~m}$ dadas por individuos principalmente de L. philippiana en el estrato dominante y $M$. planipes en el dosel intermedio (6 - $9 \mathrm{~m}$ de altura). También se presentaron individuos de S. conspicua en el borde del bosque que limita con las fajas 6,7 y 8 ( 25 a $30 \mathrm{~m}$ de altura).

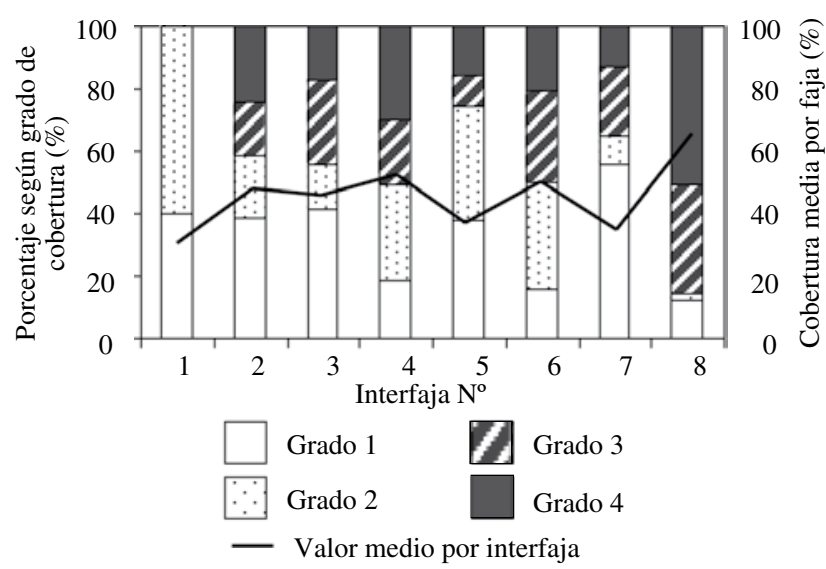

Figura 3. Porcentaje de superficie según grado de cobertura de las interfajas caracterizadas, con ancho de $3 \mathrm{~m}$ desde el borde noroeste de las interfajas. Grado 1: 0 a 25\%; grado 2: 25 a 50\%; grado 3: 50 a $75 \%$; grado 4: 75 a $100 \%$.

Percentage of the area according to the cover of the thicket between strips located towards the North-West assessed in a 3 meter belt. Cover classes: $1: 0-25 \% ; 2: 25-50 \% ; 3: 50-75 \% ; 4: 75-100 \%$.

Sobrevivencia. Coigüe en todas las fajas superó el $90 \%$ de sobrevivencia, avellano presentó el resultado más bajo en la faja 3 con un $14 \%$ y el más alto en la faja 7 , cercano al $85 \%$; por su parte, raulí, en las dos fajas en que se estableció, obtuvo sobrevivencia de 55 a $60 \%$ (figura 4).

Calidad de las plantas. En las plantas de coigüe y raulí predominó la calidad 2 con un $61,3 \%$ y $52,5 \%$, respectivamente. En avellano predominó la calidad 3 con un $40,6 \%$ de las plantas. Sólo coigüe para el factor grado de cobertura presentó diferencias significativas en la calidad de las plantas. Esto se manifestó en las fajas 7 y 8 en donde las plantas con una cobertura menor a $25 \%$ presentaron una mejor calidad $(P<0,01)$. 


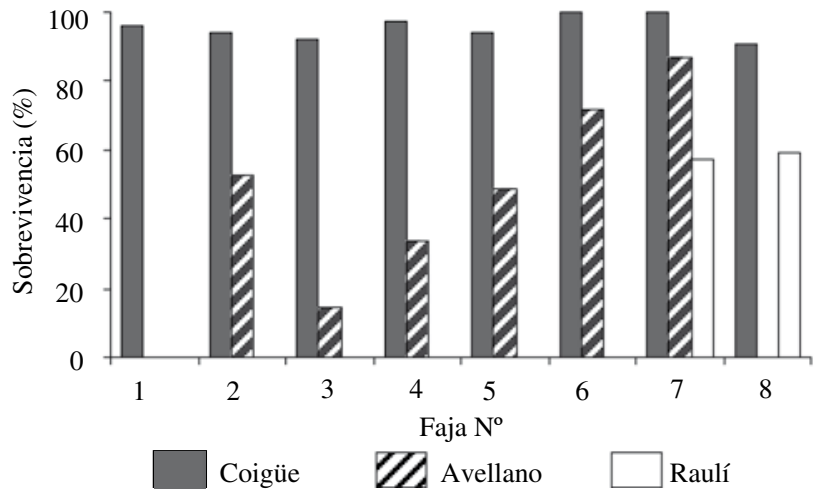

Figura 4. Porcentaje de sobrevivencia después de cuatro años por especie según faja.

Survival after 4 years (\%) per species and strip number.

Incremento en altura y diámetro de cuello. Coigüe registró el valor más alto en el IMA en altura $(0,64 \pm 0,19 \mathrm{~m})$ y diámetro de cuello $(1,08 \pm 0,31 \mathrm{~cm})$ de las tres especies evaluadas. Raulí alcanzó valores de IMA en altura de 0,54 $\pm 0,18 \mathrm{~m}$, y de diámetro de cuello $0,51 \pm 0,21 \mathrm{~cm}$; para avellano estos valores fueron, respectivamente, de $0,31 \pm$ $0,12 \mathrm{~m}$ y $0,63 \pm 0,22 \mathrm{~cm}$.

El IMA en altura de las plantas de coigüe fue superior en la faja 7, comparado con todas las demás fajas $(P<0,05)$. Raulí no presentó diferencias significativas entre fajas y avellano presentó un IMA significativamente mayor en la faja 7 respecto de la faja $2(P<0,05)$ (figura 5$)$.

El IMA en diámetro de cuello de las plantas de coigüe fue significativamente mayor en las fajas $1,2,3,4$ y 7 , comparado con las fajas 5,6 y $8(P<0,05)$. En raulí el IMA en diámetro de cuello fue significativamente mayor en la faja 7 respecto de la 8 , y en avellano fue mayor en la faja 7 respecto de la faja $2(P<0,05)$ (figura 5).

Análisis de los factores en el crecimiento en altura y diámetro de cuello. El factor grado de cobertura presentó diferencias significativas en IMA en altura y diámetro de cuello para la especie coigüe, creciendo más las plantas con un porcentaje de cobertura menor a 50\%. Asimismo, la distancia al borde del bosque influyó significativamente en el IMA en altura y diámetro de cuello de la especie coigüe, creciendo más las plantas establecidas a más de $30 \mathrm{~m}$ del borde del bosque en las fajas 1 a 6 y en la zona media de las fajas 7 y 8 . Finalmente, el grado de cobertura influyó significativamente en el IMA en diámetro de cuello de las plantas de raulí, creciendo más las plantas bajo una cobertura menor a $25 \%$ (cuadro 2 ).

Análisis espacial. El mayor IMA en altura y diámetro de cuello de coigüe se asoció al sector alto y medio de las fajas 1 y 2, respectivamente (figura 6). Esto se corroboró por el valor de correlación entre las variables IMA en altura y diámetro de cuello $(r=0,62 ; P<0,01)$. Al relacionar a través de la gráfica el IMA tanto en altura como en diámetro de cuello con el grado de cobertura presente en la interfaja, se apreció una mayor respuesta en sectores donde el porcentaje de cobertura era menor al 50\% (figuras 3 y 6). Para las plantas de raulí establecidas en las fajas 7 y 8 se identificaron como áreas de mayor crecimiento en altura y diámetro de cuello a la zona media de la faja 7 y baja de la faja 8. Al igual que en el caso del crecimiento de coigüe, esta similar distribución se asocia a un alto valor de correlación entre el IMA en altura y diámetro de cuello $(r=0,70 ; P<0,01)$. Para raulí los IMA en altura y en diámetro de cuello no presentaron relación con el grado de cobertura presente en la interfaja (figura 6).
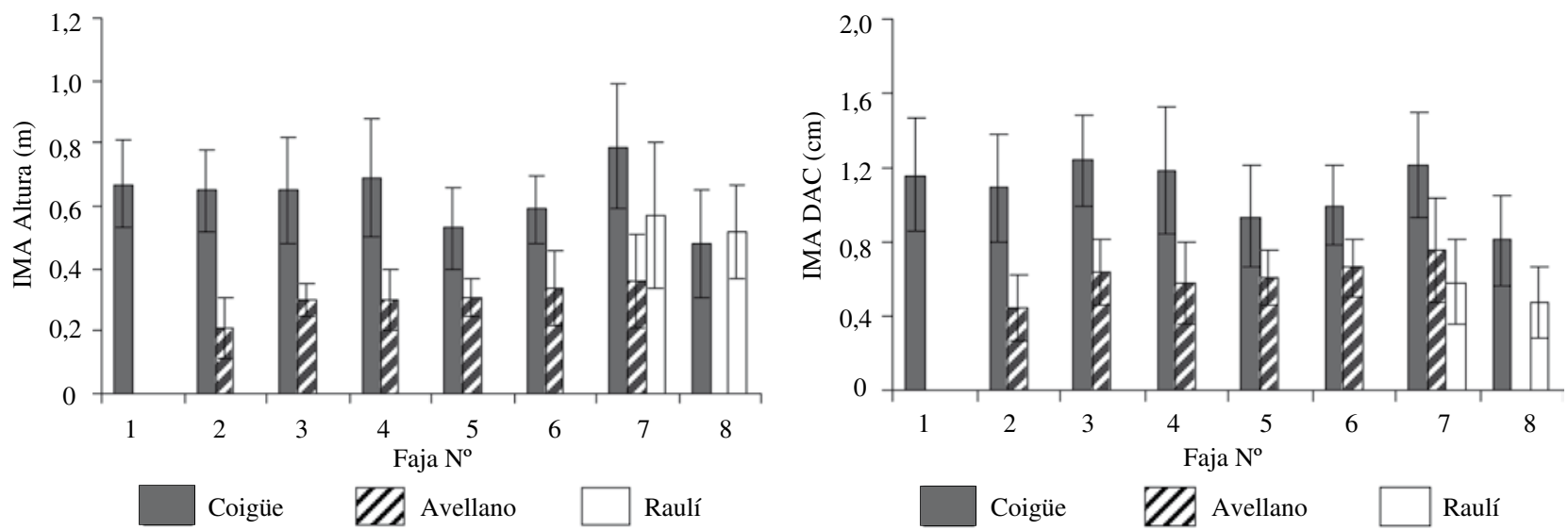

Figura 5. Incremento medio anual en altura y DAC por fajas de las tres especies evaluadas. Las barras indican \pm desviación estándar.

Mean annual increment in height and diameter per species and strip number. Bars indicate standard deviations. 
Cuadro 2. Resumen del análisis de covarianza, análisis de varianza y prueba $\mathrm{t}$ de Student independiente. Valores $\mathrm{F}$ de los factores grado de cobertura (Gr cob), distancia a la interfaja (Dist Inter) y distancia al borde del bosque (Dist bb).

Summary of the co-variance analysis and independent $t$ test. $F$ values for crown cover class (Gr cob), distance to the thicket between strips (Dist Inter.) and distance to the forest edge (Dist bb).

\begin{tabular}{|c|c|c|c|c|c|}
\hline \multirow{2}{*}{ Especie } & \multirow{2}{*}{ Factor } & \multicolumn{2}{|c|}{ Fajas 1 a 6} & \multicolumn{2}{|c|}{ Fajas 7 y 8} \\
\hline & & Alt & DAC & Alt & DAC \\
\hline \multirow[t]{3}{*}{ Coigüe } & Gr cob & $6,93 * * *$ & $4,66^{*}$ & $15,89 * * *$ & $26,54 * * *$ \\
\hline & Dist inter & $1,95 \mathrm{~ns}$ & $1,41 \mathrm{~ns}$ & $0,75 \mathrm{~ns}$ & $0,13 \mathrm{~ns}$ \\
\hline & Dist bb & $16,40 * * *$ & $20,80 * *$ & $4,71^{*}$ & $7,88^{* *}$ \\
\hline \multirow[t]{3}{*}{ Raulí } & Gr cob & - & - & $0,55 \mathrm{~ns}$ & $4,37 * *$ \\
\hline & Dist inter & - & - & $0,27 \mathrm{~ns}$ & $0,006 \mathrm{~ns}$ \\
\hline & Dist bb & - & - & $2,33 \mathrm{~ns}$ & $2,58 \mathrm{~ns}$ \\
\hline \multirow[t]{3}{*}{ Avellano $^{1}$} & Gr cob & $0,55 \mathrm{~ns}$ & $0,24 \mathrm{~ns}$ & - & - \\
\hline & Dist inter & $0,66 \mathrm{~ns}$ & $0,01 \mathrm{~ns}$ & - & - \\
\hline & Dist bb & $2,35 \mathrm{~ns}$ & $0,03 \mathrm{~ns}$ & - & - \\
\hline
\end{tabular}

${ }^{1}$ Análisis realizado para las fajas 2 a 6. ns $=$ no significativo; *: $P<0,05 ; * *: P<0,01 ; * * *: P<0,001$. DAC = diámetro de cuello; Alt $=$ altura de la planta.

\section{DISCUSIÓN}

El análisis de los resultados obtenidos no tiene la misma solidez para las tres especies estudiadas debido a limitaciones del diseño experimental que considera a) sólo a coigüe en las ocho fajas plantadas, b) diferencia en el número de plantas establecidas por especie, y c) diferencia en el rango de distancia a la interfaja por especie (cuatro distancias a interfaja para coigüe y dos para avellano y raulí). De tal manera, el análisis de los resultados para raulí y avellano son preliminares bajo este esquema de plantación.

Coigüe. La sobrevivencia en coigüe varía entre 90 y 100\%, registrando los mayores porcentajes de las tres especies evaluadas (figura 4). Estos valores concuerdan con lo afirmado por Donoso et al. (1993), quienes plantean que coigüe, a pesar de ser una especie intolerante a la sombra, presenta una menor tasa de mortalidad debido a la eficiencia del follaje de su copa, la cual requiere una menor superficie para sobrevivir y crecer. Los resultados de sobrevivencia de este estudio son superiores a los de una plantación a campo abierto de un año de edad establecida en la Cordillera de Los Andes de la provincia de Valdivia, cercana al área de estudio, en donde la sobrevivencia fluctuó entre 65 y $70 \%$ (Donoso et al. 2007). Esto es, a su vez, superior a lo reportado en estudios realizados en la Cordillera de la Costa de la provincia de Valdivia: coigüe presentó después de dos periodos vegetativos una sobrevivencia de $70 \%$ en una plantación en fajas y $40 \%$ a campo abierto (Torres
1996); en otro estudio, después de un periodo vegetativo, la sobrevivencia de coigüe fue de $75 \%$ (Büchner 2007). En el presente trabajo a la alta sobrevivencia se suma una alta proporción de plantas de buena calidad, la que se podría mejorar aún más con una poda de formación que corrigiera el doble ápice, principal problema en la malformación de las plantas. La mejor calidad de las plantas de coigüe se presenta en condiciones de mayor luminosidad, característica propia de especie intolerante, la cual bajo condiciones de semisombra tiende a generar más de un ápice principal.

La condición de coigüe de intolerante a la sombra (Donoso 1981b, Donoso 1993) admitía suponer un mayor crecimiento de esta especie en las áreas con una mayor exposición al sol. Los resultados en IMA en altura y diámetro de cuello permiten considerar como verdadera esta hipótesis y confirmar que esta especie crece favorablemente en condiciones de mayor luminosidad (cuadro 2, figura 6). Estas características de micrositio que proporcionan insolación durante la mayor parte del día, lo que incide en una mayor temperatura del aire y del suelo (Otero et al. 1994, Barg y Edmonds 1999), estarían favoreciendo el mayor crecimiento de estas plantas. Hay que considerar que el área de estudio presenta precipitaciones durante todos los meses del año y un alto nivel de capacidad de agua aprovechable del suelo, exposición SO en la cual existe una menor incidencia del sol, lo que implica mayor humedad, razones por las cuales las plantas no presentarían riesgos de sequía durante los meses de verano. Estas buenas características de sitio cobran importancia 


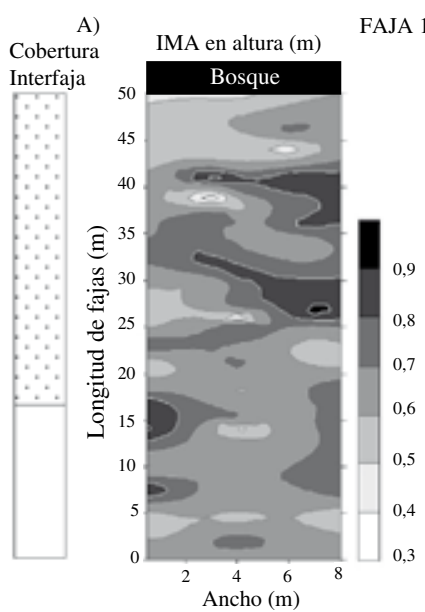

C)

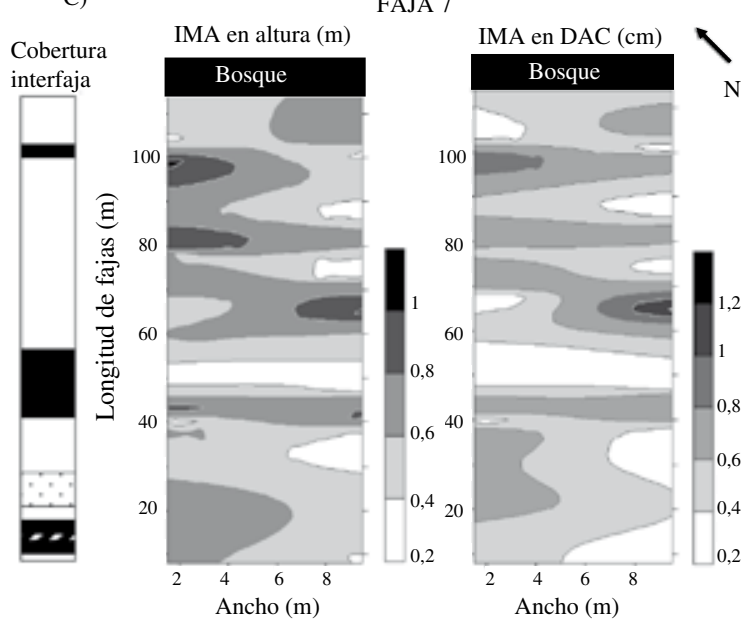

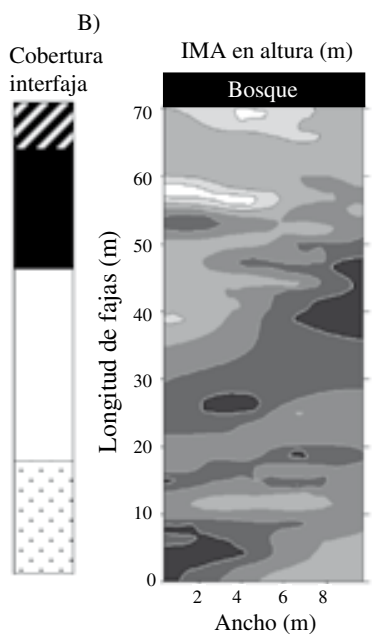

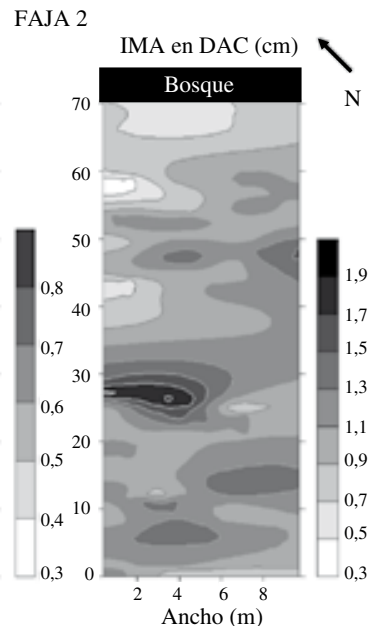

D)

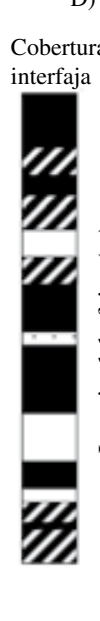

FAJA 8

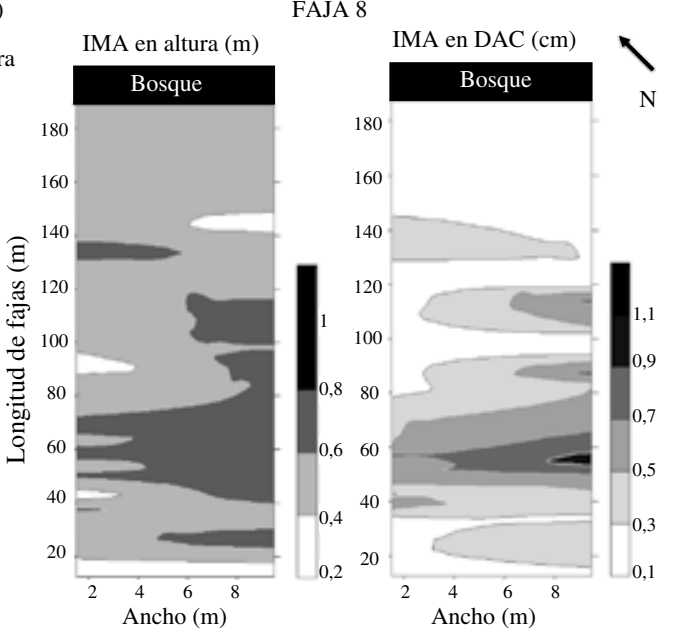

Figura 6. Incremento medio anual en altura y diámetro de cuello (DAC) de las plantas de coigüe establecidas en las fajas 1 (A) y 2 (B); raulí en las fajas 7 (C) y 8 (D). Los colores oscuros representan áreas de mayor crecimiento y corresponden al eje z. El eje y corresponde a la longitud de las fajas y tiene como origen el borde inferior de la faja, alcanzando el borde del bosque. El eje x tiene como origen el borde noroeste inferior de la faja. El borde del bosque caracterizado ubicado en el límite superior de las fajas evaluadas presenta un grado de cobertura mayor a 75\%. Los grados de cobertura de interfaja son: $\square$ grado $1(<25 \%), \because$ grado $2(25$ a $50 \%), \square$ grado 3 (50 a $75 \%)$, $\square$ grado $4(>75 \%)$.

Mean annual increment in height and in diameter at ground height for $N$. dombeyi established in strips number 1 (A) and 2 (B); N. alpina established in strips number 7 (C) and 8 (D). Dark tones indicate the areas with higher growth values in the $\mathrm{Z}$ axis. $\mathrm{Y}$ axis represents the length of the strips with its origin in the lower edge of each strip, and the higher value at the forest edge. $\mathrm{X}$ axis origin is located in the North-West lower edge of each strip. Forest edge has a crown cover $>75 \%$. Cover categories for the thicket between parallel strips are: $1: 0-25 \% ; 2: 25-50 \% ; 3: 50-75 \% ; 4: 75-100 \%$.

si se considera otro ensayo realizado en la Cordillera de la Costa de Valdivia, en donde la presencia de un estrato protector alrededor de la plantación proveía de mejores características de sitio que favorecían el desarrollo de las plantas, debido a la crítica disponibilidad de agua en el verano, a la escasa profundidad del suelo y a la exposición norte que presentaba el ensayo (Torres 1996). En particular, las fajas 1 y 2 muestran mayor IMA en altura y diámetro de cuello en las plantas ubicadas en el sector medio y bajo, con una tendencia a crecer más cuando la interfaja presenta un grado de cobertura menor a 50\%, como en el caso de la faja 1 (figura 6). Esto indicaría, al igual que en el caso de la distancia al borde del bosque, que el crecimiento se ve favorecido por la incidencia de mayor luz en las plantas. Por otro lado, el que la distancia de las plantas a la interfaja no influya en el IMA en altura y diámetro de cuello (cuadro 2), estaría indicando que la diferencia gradual en la sombra proyectada por la vegetación de interfaja y el efecto de competencia por agua y elementos nutritivos que pudiera ejercer ésta en las plantas de la hilera más cercana, no influyó en el crecimiento de la plantación (figura 6).

Coigüe en esta plantación en fajas presenta mejores y similares resultados en IMA en diámetro de cuello y 
altura, respectivamente, a los reportados por Donoso et al. (2007) al primer año, quienes registraron crecimientos de $0,69 \mathrm{~cm}$ en diámetro de cuello y $0,73 \mathrm{~m}$ en altura, y mejores resultados a los reportados por Büchner (2007), quien determinó un crecimiento de $0,45 \mathrm{~m}$ en altura durante el primer año, ambas plantaciones realizadas a campo abierto.

Raulí. Se registraron resultados sólo regulares de sobrevivencia, debido principalmente a problemas en la época de establecimiento. En ese momento raulí presentó un alto porcentaje de plantas con problemas de secamiento en a lo menos un tercio de sus hojas $(64,7 \%)$ y probablemente una alta proporción de plantas no pudieron sobrevivir en los años posteriores. No obstante, la sobrevivencia en este estudio es superior a la reportada por Quiroz (2002), quien registró un $44 \%$ de sobrevivencia en la precordillera andina de la IX Región después de tres periodos vegetativos; pero es inferior a la reportada por Grosse (1988), en la precordillera andina de la provincia de Malleco a $790 \mathrm{~m}$ s.n.m., después de un periodo vegetativo, quien encontró los mayores niveles de sobrevivencia entre 84 y $91 \%$, en una condición de semisombra. También es menor a plantaciones realizadas después de cosecha bajo cortas de protección en la precordillera andina de la X Región, las que presentaron una sobrevivencia de $80 \%$ (Quiroz 2002). Los resultados de calidad muestran una mayor proporción de plantas en la calidad 2, descalificadas principalmente por crecer con dos ápices, crecimiento que se puede rectificar con labores de poda de formación.

Por tratarse raulí de una especie semitolerante a intolerante a la sombra (Donoso 1981b) que puede aprovechar la protección natural de individuos en el dosel superior (Donoso 1993) y que presenta un nivel de tolerancia a la sombra mayor al de coigüe (Donoso et al. 1999), se esperaba que tuviera buen crecimiento bajo una condición de media a alta luminosidad con una protección vertical dada por árboles de gran envergadura presentes tanto en las interfajas como en las fajas 7 y 8 (cuadro 1). Esto se cumple en el caso del diámetro de cuello, variable que tiene un mayor crecimiento en una condición de mayor luminosidad (cuadro 2, figura 6). Tal comportamiento concuerda con lo registrado por Grosse (1988), quien reporta un mayor crecimiento de las plantas de raulí en condiciones de mayor luz. No obstante, contrastan con los obtenidos por Otero et al. (1995), quienes en una plantación con exposición norte en la Cordillera de Los Andes de la provincia de Valdivia registraron los mayores valores de crecimiento en condiciones de menor luminosidad. Esta diferencia indicaría la existencia de un efecto de la exposición en el establecimiento de plantaciones y la respuesta en crecimiento de éstas. Sin embargo, es necesaria mayor investigación que permita validar lo expuesto.

Los factores distancia a la interfaja y distancia al borde del bosque no tienen incidencia en el IMA en altura y diámetro de cuello de las plantas de raulí. Lo anterior podría estar explicado por la cobertura de copas de árboles remanentes de alto valor de área basal en las fajas e interfajas 7 y 8 (cuadro 1), lo que estaría enmascarando la importancia de los factores señalados para el crecimiento (figura 6). Sumado a esto se debería tomar en cuenta que el diseño experimental considera la plantación de raulí en las hileras laterales, lo que dificulta el análisis del factor distancia a la interfaja, debido a que existe un rango más acotado que coigüe para el estudio de este factor. El IMA en altura en este estudio resulta superior a los reportados por Otero et al. (1995), quienes en una plantación de dos años de edad registraron un incremento promedio de 0,41 y $0,36 \mathrm{~m}$ en fajas de 45 y $60 \mathrm{~m}$, respectivamente. Igualmente fue superior a resultados bajo método de protección uniforme, obtenidos por los mismos autores, quienes registraron un incremento de $0,32 \mathrm{~m}$ anuales.

Avellano. Esta especie posee condiciones tanto de intolerante como de semitolerante (Donoso 1981b) y es característica su adaptabilidad a diferentes condiciones de luminosidad (Donoso et al. 1992), lo que hacía altamente probable su adaptación y buen desarrollo en plantación en fajas. Quizás estas características le permitieron obtener buenos resultados tanto en el crecimiento en altura como en diámetro de cuello, registrando los mejores resultados bajo una condición de mayor cobertura de interfaja (figuras 3 , 4 y 5). Los resultados del IMA en altura son coincidentes con los reportados por Donoso y Cortés (1987), quienes registraron un valor de $0,2 \mathrm{~m}$ a los cuatro años de edad con plantas a raíz desnuda, en una plantación realizada en la Cordillera de la Costa de Valdivia. Esto indicaría que las condiciones de micrositio presentes en el área de estudio serían beneficiosas para el crecimiento de avellano, principalmente en las fajas en las que existe una mayor protección otorgada por la vegetación de interfaja y árboles remanentes (cuadro 1, figura 3). Sin embargo, existe una alta proporción de plantas quebradas por la nieve y una baja sobrevivencia de esta especie (figura 4). Esta situación demostraría que existieron problemas de adaptación de las plantas, debido presumiblemente a la procedencia de las semillas recolectadas a una altitud más baja que el área de estudio.

Aplicaciones en actividades silviculturales. Los resultados de sobrevivencia e IMA en altura y diámetro de cuello registrados en las plantas de coigüe en este trabajo son mejores que en plantaciones jóvenes realizadas a campo abierto (Torres 1996, Büchner 2007, Donoso et al. 2007) y en fajas (Torres 1996). Esto permite plantear que el establecimiento de coigüe bajo la técnica de plantación en fajas representa una alternativa para la restauración ecológica y productiva en bosques del tipo Coigüe-RaulíTepa degradados por tala con fines madereros. Debido a las limitaciones del diseño experimental, raulí presenta resultados menos definitivos al establecerse bajo este tipo de esquema de plantación. Sin embargo, estos resultados 
permiten considerar como muy importantes las labores que se realizan en el establecimiento de la plantación, la época y el tipo de planta a emplear, ya que los principales problemas se registraron en la etapa inicial del establecimiento. El avellano, aunque presenta buenos resultados en el crecimiento, por su baja sobrevivencia y su muy mala calidad haría necesaria la recolección de semillas a una altitud similar al área de establecimiento y su plantación bajo una fuerte protección lateral y vertical. Es el caso de la faja 7 de esta plantación, en donde presenta los mejores resultados de sobrevivencia e IMA en altura y diámetro de cuello (figuras 4,5 y 6 ).

El estudio permite constatar que al existir altos niveles de cobertura de interfaja (figura 3 ) y alto valor de área basal en árboles remanentes tanto en fajas e interfajas (cuadro 1), tal como sucede en la faja 8, el IMA en altura y diámetro de cuello de las plantas de coigüe y raulí decrecen fuertemente (figuras 5 y 6). Esto ocurre como consecuencia principalmente de la falta de luz, situación que también se ve reflejada en la calidad de las plantas de coigüe. Por esto se plantea como alternativa, en áreas donde existan árboles de gran envergadura, la corta de algunos ejemplares que permitan una mayor entrada de luz, en el caso de elegir especies intolerantes para la plantación en fajas.

Recomendaciones. Coigüe presenta los mayores valores de sobrevivencia (figura 4), IMA en altura y diámetro de cuello, por lo que se recomienda el establecimiento de esta especie en fajas para futuras plantaciones. Los tratamientos efectuados para el control de la competencia en esta plantación resultaron beneficiosos para el crecimiento de las especies establecidas. Esto hace recomendable el tipo de actividades de manejo llevadas a cabo en esta plantación. Las diferencias de crecimiento fueron significativas en el factor distancia al borde del bosque, siendo indiferente a la distancia a la interfaja (cuadro 2), por lo que se recomienda aumentar el ancho de las fajas en futuras plantaciones experimentales, que permitan concluir sobre el ancho óptimo de faja para desarrollar al máximo el potencial de crecimiento de coigüe. En futuros diseños experimentales se recomienda plantar raulí en fajas considerando interfajas constituidas principalmente de vegetación arbustiva y un rango más amplio de distancias a las mismas, que permitan concluir con mayor claridad sobre la incidencia de los factores distancia a la interfaja y grado de cobertura vegetal en el crecimiento. Además, se recomienda la evaluación de este diseño de plantación con otras especies y en otra exposición, que permita comparar la relación del crecimiento de las plantas con respecto a la vegetación remanente entre fajas.

\section{AGRADECIMIENTOS}

Este estudio se realizó con el apoyo del proyecto CRN 03 del Inter American Institute for Global Change
Research (IAI) y al Núcleo Científico Milenio FORECOS (MIDEPLAN), proyecto P04-065-F. Se agradece a Rodrigo Becerra y a don Juan Ávila por su valiosa colaboración en la recolección de datos en San Pablo de Tregua.

\section{REFERENCIAS}

Barg A, R Edmonds. 1999. Influence of partial cutting on site microclimate, soil nitrogen dynamics, and microbial biomass in Douglas-fir stands in western Washington. Canadian Journal Forest Research 29: 705-713.

Büchner C. 2007. Respuesta inicial de una plantación de Nothofagus dombeyi a distintas dosis de fertilizantes, en la Precordillera de la Costa de Valdivia. Tesis Ingeniero Forestal. Valdivia, Chile. Facultad de Ciencias Forestales, Universidad Austral de Chile. 33 p.

CIREN (Centro de Investigación de Recursos Naturales, CL). 2001. Estudio agrológico X Región. Descripciones de suelos materiales y símbolos. Tomo 1. Santiago, Chile. Centro de Investigación de Recursos Naturales. 199 p.

Donoso C. 1981a. Tipos forestales de los bosques nativos de Chile. Investigación y desarrollo forestal. Santiago, Chile. CONAF, FAO, FO: DP/CHI/76/003. Corporación Nacional Forestal. 83 p. (Documento de Trabajo $\mathrm{N}^{\circ} 38$ ).

Donoso C. 1981b. Ecología Forestal. El bosque y su medio ambiente. Santiago, Chile. Universitaria. 369 p.

Donoso C. 1993. Bosques templados de Chile y Argentina; variación, estructura y dinámica. Santiago, Chile. Universitaria. $477 \mathrm{p}$.

Donoso C, M Cortés. 1987. Semillas y técnicas de vivero y plantaciones para especies de los tipos forestales de la X Región. Informe de Convenio No 134 . UACh-CONAF. Valdivia, Chile. Universidad Austral de Chile. 21 p.

Donoso C, B Escobar, M Cortés. 1992. Técnicas de vivero y plantaciones para avellano (Gevuina avellana). Documento Técnico 63. Chile Forestal (196): 7 p.

Donoso P, T Monfil, L Otero, L Barrales. 1993. Estudio de crecimiento de plantaciones y renovales manejados de especies nativas en el área andina de las provincias de Cautín y Valdivia. Ciencia e investigación Forestal 7(2): 253-287.

Donoso P, M González, B Escobar, I Basso, L Otero. 1999. Viverización y plantación de raulí, roble y coigüe en Chile. Capítulo 7. In Donoso C, A Lara eds. Silvicultura de los bosques nativos de Chile. Universitaria. Santiago, Chile. p. 177-244.

Donoso P, V Gerding, D Uteau, D Soto, O Thiers, C Donoso. 2007. Efecto de la fertilización y cobertura de malezas en el crecimiento inicial y la mortalidad de una plantación de Nothofagus dombeyi en la Cordillera de Los Andes. Bosque 28 (3): 249-255.

Golden Software Inc. 1999. Surfer: a powerful contouring, gridding and surface mapping package for Scientists and Engineers. Consultado 10 de febrero 2006. Disponible en http://www.goldensoftware.com.

González M, C Donoso, S Fraver. 1997. Respuesta inicial de Eucryphia cordifolia Cav., Laurelia sempervirens R. et $\mathrm{P}$. Tul. y Aextoxicon punctatum R. et P. en plantaciones mixtas en sectores recientemente florecidos con Chusquea quila Kunth en el centro sur de Chile. Bosque 18(1): 53-59. 
Grosse H. 1988. Crecimiento de plantaciones con Raulí y Roble bajo dosel en dependencia del grado de luminosidad y fertilización. Ciencia e Investigación Forestal 5(2): 13-29.

Hernández I, A Vita. 2004. Reforestación para la expansión de olivillo. Capítulo 18. In Squeo F, J Gutiérrez, I Hernández eds. Historia Natural del Parque Nacional Fray Jorge. La Serena (Chile). La Serena, Chile. Ediciones Universidad de La Serena. p. 307-319.

Lara A, A Altamirano, O Thiers, A Tacón. 2002. Plan de Manejo San Pablo de Tregua. Valdivia, Chile. Universidad Austral de Chile. 66 p.

Mella A, A Kühne. 1985. Sistemática y descripción de las familias, asociaciones y series de suelos derivados de materiales piroclásticos de la zona central-sur de Chile. Capítulo 8 . In Tosso J ed. Suelos volcánicos de Chile. Santiago, Chile. Instituto de Investigaciones Agropecuarias. Ministerio de Agricultura. p. 549-716.

Otero L, A Contreras, L Barrales. 1994. Efectos ambientales de diferentes tipos de cortas en bosque nativo, el caso de las cortas de protección en fajas. Ciencia e Investigación Forestal 8(1): 88-118.

Otero L, L Barrales, A Contreras, I Ojeda. 1995. Crecimiento inicial de plantaciones de raulí (Nothofagus alpina), en los métodos de protección en faja y protección uniforme en el área de Neltume y Coñaripe. Ciencia e Investigación Forestal 9(2): 178-189.

Quiroz I. 2002. Técnicas silvícolas y genéticas para cuatro especies nativas de interés comercial. Consultado el 17 de agosto 2006. Disponible en http://www.infor.cl.

Tacón A, J Palma. 2006. Experiencias de uso sustentable. La comercialización de los productos forestales no madereros: una oportunidad para el manejo comunitario y la valoración del bosque nativo. Capítulo 3. In Catalán R, P Wilken, A Kandzior, D Tecklin, H Burschel eds. Bosques y comunidades del sur de Chile. Santiago, Chile. Universitaria. p. 253-266.

Torres A. 1996. Grados de cobertura y fertilización en el establecimiento de plantaciones de roble (Nothofagus obliqua (Mirb.) B1.), raulí (Nothofagus alpina (Poep. et Endl.) y coigüe (Nothofagus dombeyi (Mirb.) Oerst.). Tesis Ingeniero Forestal. Valdivia, Chile. Facultad de Ciencias Forestales, Universidad Austral de Chile. 64 p.

Weinberger P, C Ramírez. 2001. Microclima y regeneración natural de raulí, roble y coigüe (Nothofagus alpina, N. obliqua y N. dombeyi). Bosque 22(1): 11-26. 